\title{
A NEW HISTOLOGICAL METHOD FOR THE STUDY OF NERVE-TRACTS IN THE BRAIN AND SPINAL CHORD.
}

BY DB. BIGM. FBEUD,

Asridant Physioian to the Vienna Gonoral Hospital.

Is the course of my studies on the structure and development of the medulla oblongata I succeded in working out the following method, which will be found a powerful aid in tracing the course of fibres in the central nervous system of the adult and the embryo.

Pieces of the organ are hardened in bichromate of potash,

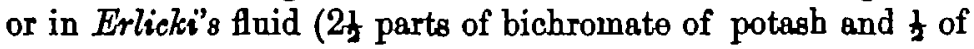
sulphate of copper to 100 parts of water), and the process of hardening is finished by placing the specimen in alcohol.; thin sections are cut by means of a microtome and washed in distilled water. The washed sections are brought into an aqueous solution of chloride of gold (1 to 100) to which is added half or an equal volume of strong alcohol. This mixture is to be preferred to the simple aqueous solution of chloride of gold, which has been hitherto used in staining preparations; the sections are to remain in it from 3 to 5 hours. With the aid of a wooden rod (metal to be avoided) they are taken out of this solution, washed in distilled water and placed in a concentrated solution of caustic soda ( 1 to 5 or 6 of water); which very soon renders them transparent and slippery. After 2 or 3 minutes the preparations are taken out of the snda with the same wooden rod (toothpick or match), and the superfluous soda is allowed to drop off. The sections are then, with the soda they still contain, put into a 10 per cent. solution of iodide of potash where they almost immediately receive a tender rose-colouring, which changes into darker hues of red during the next'5 to 15 
minutes. Now if the preparation be from the nerrous system of the adult, it may be simply washed in water and transferred into alcohol, to be mounted in the usual way. But if it be of the brain or spinal cord of the new-born or the embryo, this treatment would spoil the section, by causing it to shrink, and throwing it into folds. Therefore these preparations must be brought upon a glass-slide by means of a camel's-hair brush, and dried by gently (withont pressure) covering it with a piece of filter-paper. If the sections be very thin and soft, even this must be avoided; for the fibres of the peper would leave traces on the surface of the sections, and render them unfit for the study of the nerrous elemients. Nothing else can be done but to apply a piece of tilter-paper to the edges of the stained preparation lying on the slide, and to draw off the alkaline fluid in this way. This is by far the most tedious stage of the process, yet it is always possible to avoid shrinking and to preserve the most sensitive preparations. The sections nearly dried are allowed to remain in water a few minutes, and are then treated in the way mentioned above.

This method will never fail (as all methods of staining by chloride of gold will do) if the specimen be not overhardened and brittle, as it is sture to bo if kept in the hardening fluid an inordinate length of time. In order to stain the central nervous system of the adult, the method just described may be modified in many ways; but satisfactory preparations from the new-born or the embryo will require scrupulous attention to the details mentioned.

By this method the fibres are made to show in a pink, deep parple, blue or even black colour, and are brought distinctly into view, while the grey substance, vessels and neuroglia, lost in the slightly tinged background, are not obtruded upon the attention of the observer. A good many fine fibres, which cannot be revealed by carmine, and were not known until the methods of Exmer and Weigert came into use, are seen scattered every where throughout the white and grey substance. In the adult the big nerve-cells also appear, and the ensemble of flbres is much too complicated for analysis; in the new-born and the embryo, the nerve-fibres alone are strikingly brought out, and those bundles, which are already possessed of a 
88 NERVE-TRACTS IN THE BRAIN AND SPINAL CORD.

medullary sheatb, are distinguished by darker colouring from the others. Examined under higher powers, the single axiscylinders are so well defined as to enable one to count their number.

While this method is not adapted to the study of the grey substance, it is believed that it will prove of great service in the study of nerve-tracts, particularly if the central nervous system of the new-born or the embryo be made the subject of investigation. 